\title{
Boundary asymptotic expansions of analytic self-maps of the unit disk
}

\author{
Vladimir Bolotnikov and Nahum Zobin
}

\begin{abstract}
A characterization of analytic self-maps of the open unit disk in terms of their Taylor coefficients is due to I. Schur. We present a boundary analog of this result.
\end{abstract}

\section{Introduction}

Analytic self-maps of the open unit disk $\mathbb{D}$ have played a prominent role in function theory beginning with the work of I. Schur [11]. Following the now standard terminology, we refer to the class of functions $f$ analytic on $\mathbb{D}$ and such that $\sup _{z \in \mathbb{D}}|f(z)| \leq 1$ as the Schur class and denote it by $\mathcal{S}$. Of course this is nothing else but the unit ball of the space $H^{\infty}(\mathbb{D})$. By the maximum modulus principle, every function $f \in \mathcal{S}$ is either an analytic map of $\mathbb{D}$ into itself or a unimodular constant function.

One of the results in [11] is the following characterization of Schur class functions in terms of their Taylor coefficients. In its formulation we will make use of the numbers

$$
\gamma_{i j}:=\left.\frac{1}{i ! j !} \frac{\partial^{i+j}}{\partial z^{i} \partial \bar{z}^{j}} \frac{1}{1-|z|^{2}}\right|_{z=z_{0}}=\sum_{\ell=0}^{\min \{i, j\}} \frac{(i+j-\ell) !}{(i-\ell) ! \ell !(j-\ell) !} \cdot \frac{z_{0}^{j-\ell} \bar{z}_{0}^{i-\ell}}{\left(1-\left|z_{0}\right|^{2}\right)^{i+j-\ell+1}}
$$

associated with a point $z_{0} \in \mathbb{D}$, and of the related matrices $\Gamma_{n}:=\left[\gamma_{i j}\right]_{i, j=0}^{n-1}$.

Also, given a sequence $\mathbf{s}=\left\{s_{j}\right\}_{j \geq 0}$ of complex numbers, we let $\mathbb{U}_{n}^{\mathbf{s}}$ be the lower triangular Toeplitz matrix given by

$$
\mathbb{U}_{n}^{\mathbf{s}}=\left[\begin{array}{cccc}
s_{0} & 0 & \ldots & 0 \\
s_{1} & s_{0} & \ddots & \vdots \\
\vdots & \ddots & \ddots & 0 \\
s_{n-1} & \ldots & s_{1} & s_{0}
\end{array}\right]
$$

Mathematics Subject Classification (2010): Primary 30D40; Secondary 30E05, 47A57.

Keywords: Angular derivatives, boundary asymptotic expansion. 
Theorem 1.1. If $f(z)=\sum_{j \geq 0} s_{j}\left(z-z_{0}\right)^{j}$ belongs to $\mathcal{S}$, so that $\mathbf{s}=\left\{s_{j}\right\}_{j \geq 0}$ is the sequence of the Taylor coefficients of $f(z)$ at $z_{0} \in \mathbb{D}$, then the matrix

$$
P_{n}=\Gamma_{n}-\mathbb{U}_{n}^{\mathbf{s}} \Gamma_{n} \mathbb{U}_{n}^{\mathbf{s *}}
$$

is positive semidefinite for all $n \geq 0$.

Conversely, if $z_{0} \in \mathbb{D}$ and the sequence $\mathbf{s}=\left\{s_{j}\right\}_{j \geq 0}$ is such that (1.2) holds for all $n \geq 0$, then the power series $\sum_{j \geq 0} s_{j}\left(z-z_{0}\right)^{j}$ extends (uniquely) to a Schur class function.

In the special case where $z_{0}=0$ (considered by I. Schur) the matrix $\Gamma_{n}$ is the identity matrix and condition (1.2) takes a particularly elegant form: the Toeplitz matrices $\mathbb{U}_{n}^{\mathrm{s}}$ are contractive for all $n \geq 0$.

The objective of this paper is to obtain a boundary analog of the above characterization of Schur. Let $\mathbb{T}$ denote the unit circle $\{z:|z|=1\}$. We will write $z \widehat{\rightarrow} t_{0}$ if a point $z \in \mathbb{D}$ approaches a boundary point $t_{0} \in \mathbb{T}$ nontangentially, and we will write $z \rightarrow t_{0}$ if $z$ approaches $t_{0}$ unrestrictedly in $\mathbb{D}$. Let us assume that a function $f$ is analytic on $\mathbb{D}$ and admits the following nontangential boundary limits at $t_{0} \in \mathbb{T}$ :

$$
f_{j}\left(t_{0}\right):=\lim _{z \rightarrow t_{0}} f^{(j)}(z) / j !=s_{j} \quad \text { for all } j \geq 0
$$

Then $f$ admits an asymptotic expansion

$$
f(z)=s_{0}+s_{1}\left(z-t_{0}\right)+\cdots+s_{N}\left(z-t_{0}\right)^{N}+O\left(\left|z-t_{0}\right|^{N+1}\right)
$$

for every $N \geq 0$ as $z$ approaches $t_{0}$ nontangentially. Equation (1.4) is written in a more compact form as

$$
f(z) \sim \sum_{j \geq 0} s_{j}\left(z-t_{0}\right)^{j}
$$

Aiming at establishing a boundary analog of Theorem 1.1, one should be aware of two essential distinctions between the interior and the boundary cases. First, the limits (1.3) do not have to exist for an $f \in \mathcal{S}$. Second, even if they exist, the function $f$ may not be defined uniquely by the asymptotic expansion (1.5) unless the series $\sum_{j \geq 0} s_{j}\left(z-t_{0}\right)^{j}$ has a positive radius of convergence.

Thus the boundary case suggests an interpolation problem: describe all Schurclass functions satisfying (1.3) (or equivalently, (1.5)). We will address this issue on a separate occasion.

Necessary and sufficient conditions for the existence of a function $f$ satisfying (1.3) are given in Theorem 1.2 below, which is the main result of the paper. To formulate the result we first introduce some notation and definitions.

First, we let $\mathcal{S}_{t_{0}}^{(n)}, n \geq 1$, denote the class of Schur functions satisfying the following higher order Carathéodory-Julia condition:

$$
f \in \mathcal{S}_{t_{0}}^{(n)} \stackrel{\text { def }}{\Longleftrightarrow} f \in \mathcal{S} \text { and } \liminf _{z \rightarrow t_{0}} \frac{\partial^{2 n-2}}{\partial z^{n-1} \partial \bar{z}^{n-1}} \frac{1-|f(z)|^{2}}{1-|z|^{2}}<\infty .
$$


The inequality in (1.6) turns out to be equivalent to the Ahern-Clark conditions (see [1], [5], and [8])

$$
\sum_{k} \frac{1-\left|a_{k}\right|^{2}}{\left|t_{0}-a_{k}\right|^{2 n}}+\int_{0}^{2 \pi} \frac{d \mu(\theta)}{\left|t_{0}-e^{i \theta}\right|^{2 n}}<\infty
$$

given in terms of the inner-outer factorization of $f$ :

$$
f(z)=\prod_{k} \frac{\bar{a}_{k}}{a_{k}} \cdot \frac{z-a_{k}}{1-z \bar{a}_{k}} \cdot \exp \left\{-\int_{0}^{2 \pi} \frac{e^{i \theta}+z}{e^{i \theta}-z} d \mu(\theta)\right\} .
$$

We define $\mathcal{S}_{t_{0}}^{(0)}$ to be $\mathcal{S}$, and we let $\mathcal{S}_{t_{0}}^{(\infty)}:=\bigcap_{n \geq 1} \mathcal{S}_{t_{0}}^{(n)}$ (observe that the inclusion $\mathcal{S}_{t_{0}}^{(n+1)} \subset \mathcal{S}_{t_{0}}^{(n)}$ is proper for every $n$ ).

Another relevant object is $W_{+}$, the Wiener algebra of power series with absolutely converging coefficients:

$$
W_{+}=\left\{f(z)=\sum_{k=0}^{\infty} b_{k} z^{k}:\|f\|_{W}:=\sum_{k=0}^{\infty}\left|b_{k}\right|<\infty\right\} .
$$

Since $\|f\|_{\infty} \leq\|f\|_{W}$, convergence in the norm of $W_{+}$implies uniform convergence, and therefore every function $f \in W_{+}$can be extended continuously to $\overline{\mathbb{D}}$. Thus, if conditions (1.3) hold for a function $f \in W_{+}$, then all the nontangential limits can be replaced by unrestricted ones.

Given a point $t_{0} \in \mathbb{T}$ and a sequence $\mathbf{s}=\left\{s_{j}\right\}_{j \geq 0}$ of complex numbers, we introduce a structured $n \times n$ matrix

(1.7) $\mathbb{P}_{n}^{\mathbf{s}}\left(t_{0}\right)=\left[p_{i j}^{\mathbf{s}}\right]_{i, j=1}^{n}=\left[\begin{array}{cccc}s_{1} & s_{2} & \ldots & s_{n} \\ \vdots & \vdots & & \vdots \\ s_{n} & s_{n+1} & \ldots & s_{2 n-1}\end{array}\right] \boldsymbol{\Psi}_{n}\left(t_{0}\right)\left[\begin{array}{ccc}\bar{s}_{0} & \ldots & \bar{s}_{n-1} \\ & \ddots & \vdots \\ 0 & & \bar{s}_{0}\end{array}\right]$

for every $n \geq 1$, where the first factor is a Hankel matrix, the third factor is an upper triangular Toeplitz matrix (in fact, the adjoint of the matrix $\mathbb{U}_{n}^{\mathbf{s}}$ from (1.1)), and where

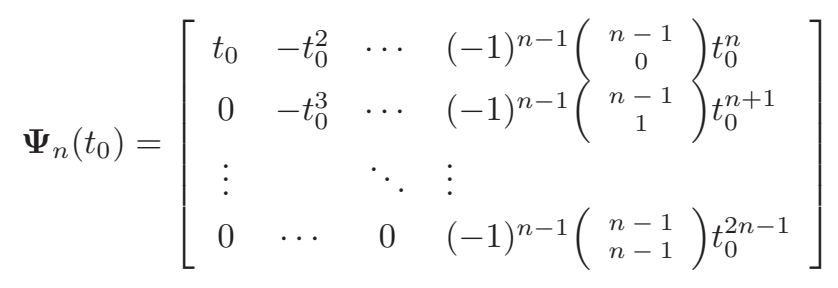

is the upper triangular matrix with the entries

$$
\Psi_{j \ell}=\left\{\begin{array}{cl}
0, & \text { if } j>\ell, \\
(-1)^{\ell-1}\left(\begin{array}{c}
\ell-1 \\
j-1
\end{array}\right) t_{0}^{\ell+j-1}, & \text { if } j \leq \ell,
\end{array} \quad(j, \ell=1, \ldots, n) .\right.
$$


The explicit formulas

$$
p_{i j}^{\mathbf{s}}=\sum_{r=1}^{j}\left(\sum_{\ell=1}^{r} s_{i+\ell-1} \Psi_{\ell r}\right) \bar{s}_{j-r} \quad(i, j \geq 1)
$$

for the entries of $\mathbb{P}_{n}^{\mathbf{s}}\left(t_{0}\right)$ follow directly from (1.8) and (1.7). Observe that the numbers $p_{i j}^{\mathbf{s}}$ depend only on $\left\{t_{0}, s_{0}, \ldots, s_{k}\right\}$ for any pair $(i, j)$ of indices such that $i+j \leq k+1$.

The main result of this paper follows:

Theorem 1.2. Given $t_{0} \in \mathbb{T}$ and $\mathbf{s}=\left\{s_{j}\right\}_{j \geq 0}$, there exists a function $f \in \mathcal{S}$ satisfying conditions (1.3) if and only if one of the following holds:

(1) $\left|s_{0}\right|<1$;

(2) $\left|s_{0}\right|=1$ and there exists an integer $n \in \mathbb{N}$ such that

$$
\mathbb{P}_{n}^{\mathbf{s}}\left(t_{0}\right)>0 \quad \text { and } \quad t_{0} \cdot\left(p_{n+1, n}^{\mathbf{s}}-\overline{p_{n, n+1}^{\mathbf{s}}}\right)>0 ;
$$

(3) $\left|s_{0}\right|=1$ and

$$
\mathbb{P}_{n}^{\mathbf{s}}\left(t_{0}\right) \geq 0 \text { for all } n \geq 1,
$$

where the matrix $\mathbb{P}_{n}^{\mathbf{s}}\left(t_{0}\right)$ and the numbers $p_{n+1, n}^{\mathbf{s}}$ and $p_{n, n+1}^{\mathbf{s}}$ are defined in $(1.7)$ and (1.9), respectively. Moreover,

(a) If $\left|s_{0}\right|<1$, then for every $\varepsilon>0$ there are infinitely many functions $f \in W_{+}$ satisfying conditions (1.3), and such that $\|f\|_{\infty}<\left|s_{0}\right|+\varepsilon$.

(b) In case (2), every function $f \in \mathcal{S}$ subject to conditions (1.3) belongs to the set $\mathcal{S}_{t_{0}}^{(n)} \backslash \mathcal{S}_{t_{0}}^{(n+1)}$. There are infinitely many such functions in $W_{+}$.

(c) In case (3), every function $f \in \mathcal{S}$, subject to (1.3), belongs to $\mathcal{S}_{t_{0}}^{(\infty)}$.

Remark 1.3. The numbers $p_{n+1, n}^{\mathbf{s}}$ and $p_{n, n+1}^{\mathbf{s}}$ are the entries of the structured matrix $\mathbb{P}_{n+1}^{\mathbf{s}}\left(t_{0}\right)$; thus the second inequality in (1.10) implies that $\mathbb{P}_{n+1}^{\mathbf{s}}\left(t_{0}\right)$ is not Hermitian. On the other hand, since the factors $\boldsymbol{\Psi}_{n}\left(t_{0}\right)$ and $\mathbb{U}_{n}^{\mathbf{s} *}$ in (1.7) are upper triangular, then for every $k<n$, the matrix $\mathbb{P}_{k}^{\mathbf{s}}\left(t_{0}\right)$ is a leading submatrix of $\mathbb{P}_{n}^{\mathbf{s}}\left(t_{0}\right)$. Therefore, the number $n$ in part (2) of Theorem 1.2 is the greatest positive integer such that the matrix $\mathbb{P}_{n}^{\mathbf{s}}\left(t_{0}\right)$ is positive definite.

Remark 1.4. The existence of a function $f \in \mathcal{S}$ satisfying condition (1.4) for a fixed $N \geq 0$ was studied in [2]. It was shown that once such a truncated problem admits a solution, it admits a rational solution as well. Although we cannot guarantee the existence of a rational solution in the present infinite context, Theorem 1.2 shows that at least in cases (1) and (2), there exist solutions from the Wiener algebra. In the course of the proof we will show that there are solutions which belong to $W_{+}$along with all their derivatives.

The outline of the paper is the following: the sufficiency of case (1) in Theorem 1.2 is justified in Section 2; the rest of the proof of the theorem is presented in Section 3. 


\section{The first sufficient condition}

Lemma 2.1. Let $\left\{b_{k}\right\}_{k \geq 1}$ be a sequence of real numbers. For every $\varepsilon>0$, there exists a function $f \in W_{+}$such that

$$
f(1)=0, \quad f^{(k)}(1)=b_{k}(k \geq 1) \quad \text { and } \quad\|f\|_{\infty}<\varepsilon .
$$

Proof. Our proof is based on a modification of a beautiful construction suggested by Emile Borel [7] in 1895, which, in turn, was seemingly inspired by a construction proving that a conditionally convergent series of real numbers can be made convergent to any given sum by a permutation of its terms (B. Riemann).

Without loss of generality we may assume that $b_{1} \neq 0$. Indeed, if the problem (2.1) has a solution for every $b_{1} \neq 0$, then we can find a function $f \in W_{+}$with $\|f\|_{\infty}<\varepsilon / 2$ satisfying the interpolation conditions (2.1) with $b_{1}=\varepsilon / 2$. Then the function $\widetilde{f}(z)=f(z)-\frac{\varepsilon}{2} \cdot z$ will satisfy the conditions $(2.1)$ with $b_{1}=0$.

Assuming that $b_{1} \neq 0$, we construct recursively

1. a decreasing sequence $\left\{c_{j}\right\}_{j \geq 1}$ of positive numbers such that

$$
\sum_{j=1}^{\infty} c_{j}=\infty \text { and } \sum_{j=1}^{\infty} \frac{c_{j}}{j}<\varepsilon
$$

2. an increasing sequence $\left\{n_{k}\right\}_{k \geq 0}$ of natural numbers, and a sequence $\left\{B_{k}\right\}_{k \geq 1}$ of nonzero real numbers such that

$$
\left|B_{k}-\operatorname{sign}\left(B_{k}\right) \sum_{j=n_{k-1}+1}^{n_{k}} c_{j}\right| \leq c_{n_{k}} \quad \text { for all } k \geq 1 .
$$

In what follows, we use the notation $\delta_{k}:=\operatorname{sign}\left(B_{k}\right)=B_{k} /\left|B_{k}\right|$. The numbers $c_{j}$ will be picked from $\left\{\frac{\varepsilon}{3 j}, \frac{\varepsilon}{2 j}\right\}$ for every $j \geq 1$. The conditions $(2.2)$ will be satisfied for any $c_{j}$ 's chosen in this way, since

$$
\sum_{j=1}^{\infty} c_{j} \geq \sum_{j=1}^{\infty} \frac{\varepsilon}{3 j}=\infty \quad \text { and } \quad \sum_{j=1}^{\infty} \frac{c_{j}}{j} \leq \sum_{j=1}^{\infty} \frac{\varepsilon}{2 j^{2}}=\frac{\pi^{2} \varepsilon}{12}<\varepsilon .
$$

In fact, we start by taking $c_{j}=\frac{\varepsilon}{3 j}$ for all $j \geq 1$, but then in the course of construction, some of $c_{j}$ 's will be modified to $\frac{\varepsilon}{2 j}$.

To start the recursion, we let $n_{0}=0$ and $B_{1}=b_{1}$. For $k=1$, we let

$$
\delta_{1}:=\operatorname{sign}\left(B_{1}\right)=\frac{B_{1}}{\left|B_{1}\right|} \quad \text { and } \quad n_{1}:=\min \left\{n \in \mathbb{N}: \sum_{j=1}^{n} c_{j} \geq\left|B_{1}\right|\right\} .
$$

Note that $n_{1}$ is well defined due to the first condition in (2.2). By the definition of $n_{1}$ we have $n_{1} \geq 1$ and

$$
\left|B_{1}-\delta_{1} \sum_{j=1}^{n_{1}} c_{j}\right| \leq c_{n_{1}}
$$


Using $n_{1}$ and $\delta_{1}$ from (2.4), define the polynomial

$$
p_{1}(z)=\delta_{1} \sum_{\ell=1}^{n_{1}} \frac{c_{\ell}}{\ell} z^{\ell}
$$

and the real number

$$
B_{2}:=b_{2}-p_{1}^{\prime \prime}(1)=b_{2}-\delta_{1} \sum_{\ell=1}^{n_{1}}(\ell-1) c_{\ell} .
$$

If $B_{2} \neq 0$, then its construction is finished. In case the expression on the righthand side of (2.5) equals zero, we modify $c_{n_{1}}$ to $c_{n_{1}}=\frac{\varepsilon}{2 n_{1}}$. This modification does not change (2.4), but now, the formula (2.5) defines a nonzero $B_{2}$. We then let

$$
\delta_{2}:=\operatorname{sign}\left(B_{2}\right)=\frac{B_{2}}{\left|B_{2}\right|} \quad \text { and } \quad n_{2}:=\min \left\{n \in \mathbb{N}: \sum_{j=n_{1}+1}^{n} c_{j} \geq\left|B_{2}\right|\right\}
$$

which completes the first step of construction. It is clear that $n_{2} \geq n_{1}+1>n_{1}$.

Let us assume that we have already chosen the numbers $B_{1}, \ldots, B_{k} \in \mathbb{R} \backslash\{0\}$ and $1 \leq n_{1}<n_{2}<\cdots<n_{k}$. Then we also have the numbers $\delta_{j}=\operatorname{sign}\left(B_{j}\right)= \pm 1$ and the sequence $\left\{c_{j}\right\}_{j \geq 1}$ where so far $c_{j}=\frac{\varepsilon}{3 j}$ for all $j$ with possible exceptions for $j \in\left\{n_{1}, \ldots, n_{k}\right\}$ where $c_{j}=\frac{\varepsilon}{2 j}$. Using the above numbers define the polynomial

$$
p_{k}(z)=\sum_{j=1}^{k} \delta_{j} \sum_{\ell=n_{j-1}+1}^{n_{j}} \frac{c_{\ell} z^{\ell}}{\ell(\ell-1) \cdots(\ell-j+1)}
$$

and let

$$
\begin{aligned}
B_{k+1} & :=b_{k+1}-p_{k}^{(k+1)}(1) \\
& =b_{k+1}-\sum_{j=1}^{k} \delta_{j} \sum_{\ell=n_{j-1}+1}^{n_{j}} \frac{\ell(\ell-1) \cdots(\ell-k) c_{\ell}}{\ell(\ell-1) \cdots(\ell-j+1)} .
\end{aligned}
$$

If the $B_{k+1}$ defined above is not zero, then its construction is finished. If the righthand side expression in (2.7) vanishes, we modify $c_{n_{k}}$ to $c_{n_{k}}=\frac{\varepsilon}{2 n_{k}}$. This modification does not affect the previous steps, and on the other hand, formula (2.7) with the modified $c_{n_{k}}$ defines a nonzero $B_{k+1}$. Observe that since $1 \leq n_{1}$ and $n_{i}<n_{i+1}$, it follows that $n_{i}>i$ and then we have for every $\ell \geq n_{j-1}+1$,

$$
\ell-j+1>n_{j-1}-(j-1)>0,
$$

so that all the denominators in (2.7) are positive. We now let

$$
\delta_{k+1}:=\operatorname{sign}\left(B_{k+1}\right) \quad \text { and } \quad n_{k+1}:=\min \left\{n \in \mathbb{N}: \sum_{j=n_{k}+1}^{n} c_{j} \geq\left|B_{k+1}\right|\right\} .
$$

By the very definition of $n_{k+1}$, we have $n_{k+1}>n_{k}$ and

$$
\left|B_{k+1}-\delta_{k+1} \sum_{j=n_{k}+1}^{n_{k+1}} c_{j}\right| \leq c_{n_{k+1}} .
$$


The construction of the sequences $\left\{c_{j}\right\},\left\{n_{k}\right\}$ and $\left\{B_{k}\right\}$ is completed. We now define the function

$$
g(z)=\sum_{j=1}^{\infty} \delta_{j} \sum_{\ell=n_{j-1}+1}^{n_{j}} \frac{c_{\ell} z^{\ell}}{\ell(\ell-1) \cdots(\ell-j+1)} .
$$

It is readily seen from $(2.9)$ and $(2.2)$ that

$$
\|g\|_{W}=\sum_{j=1}^{\infty} \sum_{\ell=n_{j-1}+1}^{n_{j}} \frac{c_{\ell}}{\ell(\ell-1) \cdots(\ell-j+1)} \leq \sum_{j=1}^{\infty} \frac{c_{j}}{j}<\varepsilon .
$$

Fix an integer $k>0$ and let $p_{k}$ be the polynomial given in (2.6) so that

$$
g(z)=p_{k}(z)+h_{k}(z)
$$

where

$$
h_{k}(z)=\sum_{j=k+1}^{\infty} \delta_{j} \sum_{\ell=n_{j-1}+1}^{n_{j}} \frac{c_{\ell} z^{\ell}}{\ell(\ell-1) \cdots(\ell-j+1)} .
$$

Since this power series converges uniformly on compact subsets of $\mathbb{D}$, we can differentiate it to get

$$
\begin{aligned}
\frac{d^{k} h_{k}(z)}{d z^{k}} & =\sum_{j=k+1}^{\infty} \delta_{j} \sum_{\ell=n_{j-1}+1}^{n_{j}} \frac{\ell(\ell-1) \cdots(\ell-k+1) c_{\ell} z^{\ell-k}}{\ell(\ell-1) \cdots(\ell-j+1)} \\
& =\sum_{j=k+1}^{\infty} \delta_{j} \sum_{\ell=n_{j-1}+1}^{n_{j}} \frac{c_{\ell} z^{\ell-k}}{(\ell-k) \cdots(\ell-j+1)} .
\end{aligned}
$$

Therefore,

$$
\begin{aligned}
\left\|\frac{d^{k} h_{k}}{d z^{k}}\right\|_{W} & =\sum_{j=k+1}^{\infty} \sum_{\ell=n_{j-1}+1}^{n_{j}} \frac{c_{\ell}}{(\ell-k) \cdots(\ell-j+1)} \leq \sum_{j=k+1}^{\infty} \frac{c_{j}}{j-k} \\
& \leq \sum_{j=k+1}^{\infty} \frac{\varepsilon}{2 j(j-k)}=\frac{\varepsilon}{2 k} \sum_{j=1}^{k} \frac{1}{j}<\frac{\varepsilon}{2 k}(1+\ln (k+1)) .
\end{aligned}
$$

Thus, $h_{k}^{(k)}$ belongs to $W_{+}$and hence $g_{k}^{(k)}$ belongs to $W_{+}$as well. In particular, $h_{k}^{(k)}$ and $g_{k}^{(k)}$ are continuous in the closed unit disk and we thus have, from (2.11),

$$
g^{(k)}(1)=p_{k}^{(k)}(1)+h_{k}^{(k)}(1) .
$$

Since, by virtue of (2.6),

$$
p_{k}(z)=p_{k-1}(z)+\delta_{k} \sum_{\ell=n_{k-1}+1}^{n_{k}} \frac{c_{\ell} z^{\ell}}{\ell(\ell-1) \cdots(\ell-k+1)}
$$


and hence, $p_{k}^{(k)}(1)=p_{k-1}^{(k)}(1)+\delta_{k} \sum_{\ell=n_{k-1}+1}^{n_{k}} c_{\ell}$, we conclude, from (2.14),

$$
g^{(k)}(1)=p_{k-1}^{(k)}(1)+\delta_{k} \sum_{\ell=n_{k-1}+1}^{n_{k}} c_{\ell}+h_{k}^{(k)}(1) .
$$

Using the definition (2.7) of $B_{k}$, we can write the last equality as

$$
g^{(k)}(1)=b_{k}-B_{k}+\delta_{k} \sum_{\ell=n_{k-1}+1}^{n_{k}} c_{\ell}+h_{k}^{(k)}(1)
$$

which together with (2.3) gives us the following estimate:

$$
\begin{aligned}
\left|g^{(k)}(1)-b_{k}\right| & \leq\left|B_{k}-\delta_{k} \sum_{\ell=n_{k-1}+1}^{n_{k}} c_{\ell}\right|+\left|h_{k}^{(k)}(1)\right| \\
& \leq c_{n_{k}}+\left\|h_{k}^{(k)}\right\|_{W}<\frac{\varepsilon}{2 k}(2+\ln (k+1))<2 \varepsilon,
\end{aligned}
$$

where the third inequality is a consequence of (2.13) and of the inequality $c_{n_{k}} \leq$ $\frac{\varepsilon}{2 n_{k}} \leq \frac{\varepsilon}{2 k}$. Furthermore, the function

$$
h(z)=\sum_{k=1}^{\infty} \frac{b_{k}-g^{(k)}(1)}{k !}(z-1)^{k}
$$

is entire due to (2.15). Therefore $h \in W_{+}$. We now conclude from (2.16) and (2.15) that for every $z \in \overline{\mathbb{D}}$,

$$
|h(z)| \leq \sum_{k=1}^{\infty} \frac{\left|b_{k}-g^{(k)}(1)\right| \cdot 2^{k}}{k !}<2 \varepsilon\left(e^{2}-1\right) \quad(z \in \overline{\mathbb{D}}) .
$$

It follows from (2.10) that

$$
|g(1)| \leq\|g\|_{W}<\varepsilon
$$

and it is readily seen from (2.16) that

$$
h(1)=0 \quad \text { and } \quad h^{(k)}(1)=b_{k}-g^{(k)}(1) \quad \text { for all } k \geq 1 .
$$

We now conclude from (2.19) that the function $f(z)=g(z)+h(z)-g(1)$ satisfies all the interpolation conditions in (2.1). Furthermore, it belongs to $W_{+}$since $g$ and $h$ do. Finally, we can construct such an $f$ with $\|f\|_{\infty}$ arbitrarily small, since by $(2.10),(2.18)$ and $(2.19)$,

$$
\|f\|_{\infty} \leq\|g\|_{W}+\|h\|_{\infty}+|g(1)|<\varepsilon+2 \varepsilon\left(e^{2}-1\right)+\varepsilon=2 e^{2} \varepsilon .
$$


The next theorem proves the sufficiency of condition (1) and part (a) of the second statement in Theorem 1.2.

Theorem 2.2. Let $t_{0} \in \mathbb{T}, \varepsilon>0$ and $\mathbf{s}=\left\{s_{j}\right\}_{j \geq 0}$ be given and let us assume that $\left|s_{0}\right|<1$. Then there exist infinitely many functions $f \in W_{+}$satisfying conditions (1.3) and such that $\|f\|_{\infty}<\left|s_{0}\right|+\varepsilon$.

Proof. We assume without loss of generality that $t_{0}=1$. For every $k \geq 1$, we represent the number $s_{k}$ in the form

$$
s_{k}=b_{1 k}+i b_{2 k} \quad \text { where } \quad b_{k 1}, b_{2 k} \in \mathbb{R} .
$$

According to Lemma 2.1, we can find functions $f_{1}, f_{2} \in W_{+}$such that

$$
f_{i}(1)=0, \quad f_{i}^{(k)}(1)=b_{i k}(k \geq 1) \quad \text { and } \quad\left\|f_{i}\right\|_{\infty}<\varepsilon / 4 \quad(i=1,2) .
$$

Again by Lemma 2.1, we can find a function $h \in W_{+}$with $\|h\|_{\infty}<\varepsilon / 4$ and such that

$$
h\left(t_{0}\right)=0, \quad h^{\prime}\left(t_{0}\right)=1, \quad h^{(j)}\left(t_{0}\right)=0 \quad \text { for } j \geq 2 .
$$

Due to the second condition in $(2.21),\|h\|_{\infty}>0$. We thus can find a sequence $\left\{h_{j}\right\}_{j \geq 1}$ of nonzero functions in $W_{+}$satisfying conditions (2.21) and such that $\left\|h_{j+1}\right\|_{\infty}<\left\|h_{j}\right\|_{\infty}<\|h\|_{\infty}$. Then for every $j \geq 1$, the function

$$
f_{j}(z)=s_{0}+f_{1}(z)+i f_{2}(z)+h(z)-h_{j}(z)
$$

satisfies conditions (1.3) and $\|f\|_{\infty}<\left|s_{0}\right|+\varepsilon$. Observe that the sum of the three first terms on the right-hand side of (2.22) gives a particular solution of the interpolation problem (1.3) while the two last terms produce a solution of the corresponding homogeneous problem for every $j \geq 1$.

\section{Completion of the proof of Theorem 1.2}

The outline of the rest of the paper is the following. We first prove the sufficiency of case (2). Then we prove the sufficiency of case (3) and briefly discuss the determinacy of the problem (i.e., the existence of a unique solution) which may occur only in case (3). Then we show that in all cases other than (1), (2) and (3), the problem has no solutions, and finally, we prove parts (b) and (c) from the second statement of the theorem.

\subsection{The sufficiency of case (2)}

To justify the desired sufficiency we apply a certain Schur-type reduction. This reduction applies whenever

$$
\left|s_{0}\right|=1 \quad \text { and } \quad \mathbb{P}_{n}^{\mathbf{s}}\left(t_{0}\right)>0
$$


and leads us to an equivalent (in the sense of solvability) Schur-class problem

$$
\mathcal{E}(z) \sim \sum_{j \geq 0} \mathcal{E}_{j}\left(z-t_{0}\right)^{j}
$$

with the target sequence $\left\{\mathcal{E}_{j}\right\}_{j \geq 0}$ completely determined by the original sequence $\left\{s_{j}\right\}_{j \geq 0}$. It turns out that the inequality $t_{0} \cdot\left(p_{n+1, n}^{\mathbf{s}}-\bar{p}_{n, n+1}^{\mathbf{s}}\right)>0$ implies $\left|\mathcal{E}_{0}\right|<1$ and the result will follow from Theorem 2.2. The details are furnished below.

Assuming that the conditions (3.1) are met, we let

$$
T=\left[\begin{array}{cccc}
t_{0} & 0 & \ldots & 0 \\
1 & t_{0} & \ddots & \vdots \\
& \ddots & \ddots & 0 \\
0 & & 1 & t_{0}
\end{array}\right], \quad E=\left[\begin{array}{c}
1 \\
0 \\
\vdots \\
0
\end{array}\right], \quad M=\left[\begin{array}{c}
s_{0} \\
s_{1} \\
\vdots \\
s_{n-1}
\end{array}\right]
$$

$\left(T \in \mathbb{C}^{n \times n}\right.$ and $\left.E \in \mathbb{C}^{n}\right)$ and let $\widetilde{\mathbb{P}}$ be the positive definite matrix given by

$$
\widetilde{\mathbb{P}}:=\mathbb{P}_{n}^{\mathbf{s}}+M M^{*} .
$$

It is not hard to show that the numbers $M^{*} \widetilde{\mathbb{P}}^{-1} M$ and $E^{*} \widetilde{\mathbb{P}}^{-1} E$ are less than one. We let

$$
\alpha=\sqrt{1-M^{*} \widetilde{\mathbb{P}}-1} M, \quad \text { and } \beta=\sqrt{1-E^{*} \widetilde{\mathbb{P}}-1 E}
$$

and define the functions

$$
\begin{aligned}
& \mathbf{a}(z)=E^{*}\left(\widetilde{\mathbb{P}}-z \mathbb{P}_{n}^{\mathbf{s}}\left(t_{0}\right) T^{*}\right)^{-1} M, \\
& \mathbf{b}(z)=\beta\left(1-z E^{*}\left(\widetilde{\mathbb{P}}-z \mathbb{P}_{n}^{\mathbf{s}}\left(t_{0}\right) T^{*}\right)^{-1} T^{-1} E\right), \\
& \mathbf{c}(z)=\alpha\left(1-z M^{*} T^{*}\left(\widetilde{\mathbb{P}}-z \mathbb{P}_{n}^{\mathbf{s}}\left(t_{0}\right) T^{*}\right)^{-1} M\right), \\
& \mathbf{d}(z)=z \alpha \beta M^{*}\left(\mathbb{P}_{n}^{\mathbf{s}}\left(t_{0}\right)\right)^{-1} \widetilde{\mathbb{P}}\left(\widetilde{\mathbb{P}}-z \mathbb{P}_{n}^{\mathbf{s}}\left(t_{0}\right) T^{*}\right)^{-1} T^{-1} E .
\end{aligned}
$$

Some of their properties are recalled below.

Theorem 3.1. Let us assume that the conditions (3.1) are met and let $\mathbf{a}, \mathbf{b}, \mathbf{c}$, and $\mathbf{d}$ be defined as in (3.3)-(3.6). Then,

1. The function $\mathbf{S}=\left[\begin{array}{ll}\mathbf{a} & \mathbf{b} \\ \mathbf{c} & \mathbf{d}\end{array}\right]$ is rational and inner in $\mathbb{D}$. In particular, its entries (3.3)-(3.6) are rational Schur-class functions analytic on $\mathbb{T}$.

2. $\mathbf{a}_{j}\left(t_{0}\right):=\mathbf{a}^{(j)}\left(t_{0}\right) / j !=s_{j}$ for $j=0, \ldots, 2 n-1$.

3. The functions $\mathbf{b}$ and $\mathbf{c}$ have zero of multiplicity $n+1$ at $t_{0}$ and do not have other zeroes in $\mathbb{C}$. Furthermore,

$$
t_{0}^{2 n} \mathbf{b}_{n}\left(t_{0}\right)=(-1)^{n-1} \overline{\mathbf{c}_{n}\left(t_{0}\right)} \mathbf{d}\left(t_{0}\right) s_{0} \neq 0 .
$$

4. $\left|\mathbf{d}\left(t_{0}\right)\right|=1$ and $|\mathbf{d}(\zeta)|<1$ for every $\zeta \in \overline{\mathbb{D}} \backslash\left\{t_{0}\right\}$. 
The first three statements were proved in [4] (see Theorem 6.4 and Lemma 6.5 there). The last statement is a simple consequence of statements (1) and (3). Indeed, for every $\zeta \in \mathbb{T}$, the matrix

$$
\mathbf{S}(\zeta)=\left[\begin{array}{ll}
\mathbf{a}(\zeta) & \mathbf{b}(\zeta) \\
\mathbf{c}(\zeta) & \mathbf{d}(\zeta)
\end{array}\right]
$$

is unitary. Since $\mathbf{b}\left(t_{0}\right)=\mathbf{c}\left(t_{0}\right)=0$, then $\left|\mathbf{b}\left(t_{0}\right)\right|=1$. Since $\mathbf{b}(\zeta) \mathbf{c}(\zeta) \neq 0$ for every $\zeta \in \mathbb{T} \backslash\left\{t_{0}\right\}$, it follows that $|\mathbf{d}(\zeta)|<1$. By the maximum modulus principle, the latter inequality also holds for all $\zeta \in \mathbb{D}$.

Theorem 3.2. Given $t_{0} \in \mathbb{T}$ and $s_{0}, \ldots, s_{2 n-1}, s_{2 n} \in \mathbb{C}$, let us assume that the conditions (3.1) are met. Let $\mathbf{a}, \mathbf{b}, \mathbf{c}$, and $\mathbf{d}$ be the functions given in (3.3)-(3.6). Then a function $f$ belongs to $\mathcal{S}$ and satisfies conditions

$$
f_{j}\left(t_{0}\right):=\lim _{z \rightarrow t_{0}} f^{(j)}(z) / j !=s_{j} \quad \text { for } j=0, \ldots, 2 n
$$

if and only if it is of the form

$$
f(z)=\mathbf{a}(z)+\frac{\mathbf{b}(z) \mathbf{c}(z) \mathcal{E}(z)}{1-\mathbf{d}(z) \mathcal{E}(z)}
$$

for some function $\mathcal{E} \in \mathcal{S}$ such that

$$
\lim _{z \rightarrow t_{0}} \mathcal{E}(z)=\mathcal{E}_{0}:=\frac{\overline{\mathbf{d}\left(t_{0}\right)}\left(s_{2 n}-\mathbf{a}_{2 n}\left(t_{0}\right)\right)}{(-1)^{n-1} \bar{t}_{0}^{2 n}\left|\mathbf{c}_{n}\left(t_{0}\right)\right|^{2} s_{0}+s_{2 n}-\mathbf{a}_{2 n}\left(t_{0}\right)} .
$$

Furthermore, if $p_{n+1, n}^{\mathbf{s}}$ and $p_{n, n+1}^{\mathbf{s}}$ are the numbers determined from $t_{0}$ and $s_{0}, \ldots, s_{2 n}$ via the formula (1.9) and $\mathcal{E}_{0}$ is defined as in (3.9), then

$$
t_{0}\left(p_{n+1, n}^{\mathbf{s}}-\overline{p_{n, n+1}^{\mathbf{s}}}\right)=\frac{\left|\mathbf{c}_{n}\left(t_{0}\right)\right|^{2}\left(1-\left|\mathcal{E}_{0}\right|^{2}\right)}{\left|\overline{\mathbf{d}\left(\mathbf{t}_{\mathbf{0}}\right)}-\mathcal{E}_{0}\right|^{2}} .
$$

For the proof, see Corollary 4.4 and Lemma 4.6 in [2]. The next theorem justifies the second sufficient condition in Theorem 1.2.

Theorem 3.3. Let $t_{0} \in \mathbb{T}, \mathbf{s}=\left\{s_{j}\right\}_{j \geq 0}$ and $n \geq 1$ satisfy the conditions (1.10). Then there are infinitely many functions $f \in \mathcal{S} \cap W_{+}$subject to (1.3).

Proof. Every $f \in \mathcal{S}$ satisfying conditions (1.3) clearly meets conditions (3.7) and therefore, it is of the form (3.8) for some $\mathcal{E} \in \mathcal{S}$ subject to the boundary condition (3.9). Furthermore, since the coefficient functions $\mathbf{a}, \mathbf{b}, \mathbf{c}$, and $\mathbf{d}$ are rational functions analytic in the closed unit disk and since the transformation $\mathcal{E} \rightarrow f$ given by formula (3.8) is invertible, it follows that $f$ of the form (3.8) admits boundary limits $f_{j}\left(t_{0}\right)$ (nontangential or unrestricted) for all $j>2 n$ if and only if $\mathcal{E}$ admits boundary limits $\mathcal{E}_{j}\left(t_{0}\right)$ of the same type for all $j \geq 1$. It was shown in Theorem 4.3 of [2] that the limits $f_{2 n+1}\left(t_{0}\right), \ldots, f_{2 n+k}\left(t_{0}\right)$ are completely determined 
by $\mathcal{E}_{1}\left(t_{0}\right), \ldots, \mathcal{E}_{k}\left(t_{0}\right)$ and vice versa. Letting $k \rightarrow \infty$ we conclude that $f$ belongs to $\mathcal{S}$ and satisfies conditions (1.3) if and only if it is of the form (3.8) for some $\mathcal{E} \in \mathcal{S}$ subject to the conditions

$$
\mathcal{E}_{j}\left(t_{0}\right)=\mathcal{E}_{j} \quad(j \geq 0)
$$

where $\mathcal{E}_{0}$ is given by $(3.9)$ and all the other $\mathcal{E}_{j}$ 's are uniquely determined from $t_{0}$ and $\mathbf{s}=\left\{s_{j}\right\}_{j \geq 0}$. Recall that $\mathcal{E}_{0}$ satisfies equality (3.10) and $\mathbf{c}_{n}\left(t_{0}\right) \neq 0$.

Since $t_{0}\left(p_{n+1, n}^{\mathbf{s}}-\overline{p_{n, n+1}^{\mathbf{s}}}\right)>0$, it follows from (3.10) that $\left|\mathcal{E}_{0}\right|<1$. By Theorem 2.2, there are infinitely many functions $\mathcal{E} \in \mathcal{S} \cap W_{+}$satisfying conditions (3.11). For each such $\mathcal{E}$, the function $f$ of the form (3.8) belongs to $\mathcal{S}$ and satisfies condition (1.5). It remains to show that any such $f$ belongs to $W_{+}$.

Since $\mathbf{a}, \mathbf{b}, \mathbf{c}$, and $\mathbf{d}$ are rational Schur functions, they belong to $W_{+}$. The membership of $f$ of the form (3.8) in $W_{+}$will follow from Wiener's theorem once we have shown that

$$
1-\mathbf{d}(z) \mathcal{E}(z) \neq 0 \quad \text { for all } z \in \overline{\mathbb{D}} \text {. }
$$

Observe that the latter inequality holds for every $z \in \overline{\mathbb{D}} \backslash\left\{t_{0}\right\}$ by part (4) in Theorem 3.1. On the other hand, since $\mathbf{c}_{n}\left(t_{0}\right) \neq 0$ and since $\left|\mathbf{d}\left(t_{0}\right)\right|=1$, it follows from (3.9) that $\mathbf{d}\left(t_{0}\right) \mathcal{E}\left(t_{0}\right) \neq 1$ which completes the proof of (3.12) and therefore, of the theorem.

\subsection{The sufficiency of case $(3)$}

The next result justifying the requested sufficiency is known:

Theorem 3.4. Let $t_{0} \in \mathbb{T}$ and $\mathbf{s}=\left\{s_{j}\right\}_{j \geq 0}$ meet conditions (1.11). Then there exists an $f \in \mathcal{S}$ subject to (1.3).

Proof. If $\mathbb{P}_{n}^{\mathbf{s}}\left(t_{0}\right)>0$ for all $n \geq 1$, then the existence of $f$ subject to (1.3) was proved in [10] (see also [9]). This $f$ may be unique or not, depending on the convergence or divergence of certain series (see Theorems 4.1 and 4.3 in [10]). In the indeterminate case, the solution set admits a Nevanlinna-type linear fractional parametrization which we do not recall here.

Now let us assume that $\mathbb{P}_{n}^{\mathbf{s}}\left(t_{0}\right) \geq 0$ is singular for some $n \in \mathbb{N}$. Since $\mathbb{P}_{n}^{\mathbf{s}}\left(t_{0}\right)$ is a leading submatrix of $\mathbb{P}_{m}^{\mathbf{s}}\left(t_{0}\right)$ for all $m>n$, it follows that $\mathbb{P}_{m}^{\mathbf{s}}\left(t_{0}\right) \geq 0$ is also singular for all $m>n$. On the other hand, if $\left|s_{0}\right|=1$ and $\mathbb{P}_{n}^{\mathbf{s}}\left(t_{0}\right)$ and $\mathbb{P}_{n}^{\mathbf{s}}\left(t_{0}\right)$ are both positive semidefinite and singular, then there is a unique function $f \in \mathcal{S}$ satisfying (1.3) for $j=0, \ldots, 2 n-1$; moreover this unique $f$ is a Blaschke product of degree equal to the rank of $\mathbb{P}_{n}^{\mathbf{s}}$. Letting $n$ go to infinity, we conclude that the same finite Blaschke product $f$ of degree equal deg $f=\min _{n}\left(\operatorname{rank} \mathbb{P}_{n}^{\mathbf{s}}\left(t_{0}\right)\right)$ satisfies conditions (1.3) for all $j \geq 0$. 


\subsection{Complementary cases}

Let us now assume that none of the cases (1)-(3) listed in Theorem 1.2 applies to the given data $t_{0} \in \mathbb{T}$ and $\mathbf{s}=\left\{s_{j}\right\}_{j \geq 0}$. Then we have exactly one of the following four cases:

(4) $\left|s_{0}\right|>1$;

(5) $\left|s_{0}\right|=1$ and $\mathbb{P}_{1}^{\mathbf{s}}\left(t_{0}\right)=s_{1} t_{0} \bar{s}_{0} \nsupseteq 0 ;$

(6) $\left|s_{0}\right|=1$ and there is an integer $n \geq 1$ such that

$$
\mathbb{P}_{n}^{\mathbf{s}}\left(t_{0}\right) \geq 0, \quad \operatorname{det} \mathbb{P}_{n}^{\mathbf{s}}\left(t_{0}\right)=0 \quad \text { and } \quad \mathbb{P}_{n+1}^{\mathbf{s}}\left(t_{0}\right) \nsupseteq 0 ;
$$

(7) $\left|s_{0}\right|=1$ and there is an integer $n \geq 1$ such that

$$
\mathbb{P}_{n}^{\mathbf{s}}\left(t_{0}\right)>0, \quad \mathbb{P}_{n+1}^{\mathbf{s}}\left(t_{0}\right) \nsucceq 0 \quad \text { and } \quad t_{0} \cdot\left(p_{n+1, n}^{\mathbf{s}}-\overline{p_{n, n+1}^{\mathbf{s}}}\right) \leq 0,
$$

where the matrices $\mathbb{P}_{n}^{\mathbf{s}}\left(t_{0}\right), \mathbb{P}_{n+1}^{\mathbf{s}}\left(t_{0}\right)$ and the numbers $p_{n+1, n}^{\mathbf{s}}, p_{n, n+1}^{\mathbf{s}}$ are defined via formulas (1.7) and (1.9).

As we already mentioned, the solvability criterion for the truncated problem (1.4) for a fixed $N \geq 0$ was established in [2]. It follows from this criterion that all the cases (4)-(7) above fall into the "negative" side: in each case there is an $N \geq 0$ such that the boundary conditions

$$
f_{j}\left(t_{0}\right):=\lim _{z \rightarrow t_{0}} f^{(j)}(z) / j !=s_{j} \quad(j=0, \ldots, N)
$$

are satisfied by no Schur-class function $f$. In case (4), $N=0$ since $s_{0}$ cannot be equal to the boundary limit of an $f \in \mathcal{S}$ by the very definition of the class $\mathcal{S}$. In case (5), $N=1$ by the Carathéodory-Julia theorem. In cases (6) and (7), $N=2 n+3$; we refer to [2] for more details.

In any event, in each of the cases (4)-(7), the infinite problem (1.3) has no Schur-class solutions. Since the cases (4)-(7) are complementary to the cases (1)-(3) in Theorem 1.2, and all together cover all the possibilities, the "only if" part in the first statement of Theorem 1.2 follows.

\subsection{The boundary behavior of solutions}

The functions of the class $\mathcal{S}_{t_{0}}^{(n)}$ defined in (1.6) can be characterized in terms of their boundary angular derivatives as follows:

Theorem 3.5. Let us assume that we are given a function $f \in \mathcal{S}$, a point $t_{0} \in \mathbb{T}$ and an integer $n \in \mathbb{N}$. Then $f$ belongs to the class $\mathcal{S}_{t_{0}}^{(n)}$ if and only if the nontangential boundary limits $f_{j}\left(t_{0}\right)$ exist for $j=0, \ldots, 2 n-1$ and satisfy

$$
\left|f_{0}\left(t_{0}\right)\right|=1 \quad \text { and } \quad \mathbb{P}_{n}^{f}\left(t_{0}\right) \geq 0,
$$

where the matrix $\mathbb{P}_{n}^{f}\left(t_{0}\right)$ is defined by

$$
\mathbb{P}_{n}^{f}\left(t_{0}\right)=\left[\begin{array}{ccc}
f_{1}\left(t_{0}\right) & \ldots & f_{n}\left(t_{0}\right) \\
\vdots & & \vdots \\
f_{n}\left(t_{0}\right) & \ldots & f_{2 n-1}\left(t_{0}\right)
\end{array}\right] \boldsymbol{\Psi}_{n}\left(t_{0}\right)\left[\begin{array}{ccc}
\overline{f_{0}\left(t_{0}\right)} & \ldots & \overline{f_{n-1}\left(t_{0}\right)} \\
& \ddots & \vdots \\
0 & & \overline{f_{0}\left(t_{0}\right)}
\end{array}\right]
$$


The proof can be found in [3]. Observe that $\mathbb{P}_{n}^{f}\left(t_{0}\right)$ has the same structure as the matrix $\mathbb{P}_{n}^{\mathbf{s}}\left(t_{0}\right)(1.7)$, but is constructed from the boundary limits $f_{j}\left(t_{0}\right)$ of $f$ rather than from arbitrary parameters $s_{j}$.

Corollary 3.6. Let us assume that $f \in \mathcal{S}$ satisfies conditions (1.3) for given $t_{0} \in \mathbb{T}$ and $\mathbf{s}=\left\{s_{j}\right\}_{j \geq 0}$.

1. If conditions (1.11) are met, then $f$ belongs to $\mathcal{S}_{t_{0}}^{(\infty)}$.

2. If conditions (1.10) are met, then $f$ belongs to $\mathcal{S}_{t_{0}}^{(n)} \backslash \mathcal{S}_{t_{0}}^{(n+1)}$.

Proof. Since $f$ satisfies conditions (1.3), we obviously have $f_{0}\left(t_{0}\right)=s_{0}$ and $\mathbb{P}_{n}^{f}\left(t_{0}\right)=$ $\mathbb{P}_{n}^{\mathbf{s}}\left(t_{0}\right) \geq 0$ for every $n \geq 1$. Thus conditions (1.11) tell us that $f\left(t_{0}\right)|=| s_{0} \mid=1$ and $\mathbb{P}_{n}^{f}\left(t_{0}\right) \geq 0$ for every $n \geq 0$. By Theorem 3.5, $f$ belongs to $\mathcal{S}_{t_{0}}^{(n)}$ for every $n \geq 1$ and thus, $f \in \mathcal{S}_{t_{0}}^{(\infty)}$ which completes the proof of part (1). In case the conditions (1.10) are in force, we again invoke Theorem 3.5 to conclude that $f$ belongs to $\mathcal{S}_{t_{0}}^{(n)}$ and does not belong to $\mathcal{S}_{t_{0}}^{(n+1)}$.

\subsection{Conclusion}

The following summary shows that the proof of Theorem 1.2 is completed. Indeed, the "if" part of the first statement (that is, the sufficiency of conditions (1)-(3)) was established in Theorems 2.2, 3.3 and 3.4, while the "only if" part was justified in Subsection 3.3. Part (a) of the second statement is covered by Theorem 2.2 and statements (b) and (c) are covered by Theorem 3.3 and Corollary 3.6.

\section{References}

[1] Ahern, P. R. And Clark, D. N.: Radial limits and invariant subspaces. Amer. J. Math. 92 (1970) 332-342.

[2] Bolotnikov, V.: On higher order boundary derivatives of an analytic self-map of the unit disk. J. Approx. Theory 163 (2011), no. 4, 568-589.

[3] Bolotnikov, V. And Kheifets, A.: A higher order analogue of the CarathéodoryJulia theorem. J. Funct. Anal. 237 (2006), no. 1, 350-371.

[4] Bolotnikov, V. And Kheifets, A.: The higher order Carathéodory-Julia theorem and related boundary interpolation problems. In Recent advances in matrix and operator theory, 63-102. Oper. Theory Adv. Appl. 179, Birkhäuser, Basel, 2008.

[5] Bolotnikov, V. And Kheifets, A.: Carathéodory-Julia type theorems for operator valued Schur functions. J. Anal. Math. 106 (2008), 237-270.

[6] Bolotnikov, V. And Kheifets, A.: Carathéodory-Julia type conditions and symmetries of boundary asymptotics for analytic functions on the unit disk. Math. Nachr. 282 (2009), no. 11, 1513-1536.

[7] Borel, E.: Sur quelques points de la théorie des fonctions. Ann. Sci. École Norm. Sup. (3) 12 (1895), 9-55.

[8] Fricain, E. And Mashreghi, J.: Boundary behavior of functions in the De Branges-Rovnyak spaces. Complex Anal. Oper. Theory 2 (2008), no. 1, 87-97. 
[9] Kheifets, A.: Hamburger moment problem: Parseval equality and $A$-singularity. J. Funct. Anal. 141 (1996), no. 2, 374-420.

[10] Kovalishina, I. V.: A multiple boundary value interpolation problem for contracting matrix functions in the unit disk. J. Soviet Math. 52 (1990), no. 6, 3467-3481.

[11] Schur, I.: Über Potenzreihen, die im Innern des Einheitskreises beschränkt sind. I. J. Reine Angew. Math. 147 (1917), 205-Â232.

Received May 23, 2011.

Vladimir Bolotnikov: Department of Mathematics, College William and Mary, Williamsburg, Virginia 23187-8795, USA.

E-mail: vladi@math.wm.edu

Nahum Zobin: Department of Mathematics, College William and Mary, Williamsburg, Virginia 23187-8795, USA.

E-mail: nxzobi@wm.edu

The first author was partially supported by the NSF Grant DMS 0901124. 